\title{
A economia brasileira puxada pela demanda agregada
}

\author{
JOSÉ LUIS OREIRO \\ LUCIANO NAKABASHI \\ GUSTAVO JOSÉ DE GUIMARÃES E SOUZA*
}

The Brazilian economy pulled by the aggregate demand. This article aims to present the demand-led growth theory and some empirical evidences for a demand-led growth regime in Brazil. First of all, we will do a brief review of the theory of demand led-growth, based in the seminal work of Kaldor (1988), for whom long-run growth is determined by the growth rate of consumption expenditures and the growth rate of exports. Based in the empirical methodology developed by Atesoglu (2002), we run some econometric tests for the hypothesis of demand-led growth for Brazilian economy. The results of such tests shown that near of $85 \%$ of GDP growth in Brazil in the period 1991-2005 is explained by variables at the demand side of the economy. Besides that, based in the methodology developed by Ledesma and Thirwall (2002), we shown that natural rate of growth for Brazilian economy is endogenous, increasing during boom times. This means that appears to be no restrictions in the supply side of the economy for a faster growth of Brazilian economy. Finally, we argue that a necessary condition for a sustained growth of Brazilian economy is the adoption of a export-led growth model. For such it is necessary to put an end on the actual over-valuation of real exchange rate

Keywords: economic growth; aggregate demand; real exchange rate.

JEL Classification: O11; O47; O54.

\footnotetext{
* Respectivamente, Professor do Departamento de Economia da Universidade de Brasília, Pesquisador nível I do CNPq. E-mail: joreiro@unb.br; Professor do Departamento de Economia da UFPR e Pesquisador do CNPQ. E-mail: Luciano.nakabashi@gmail.com; Professor da Universidade Católica de Brasília. E-mail: gustavojgs@gmail.com. Submetido: Abril 2007; Aprovado: Junho 2009.
} 


\section{INTRODUÇÃO}

Nos últimos 20 anos a economia brasileira teve um crescimento médio anual de apenas $0,7 \%$ em termos per capita, valor bastante inferior daquele observado entre 1950 e 1980, situado em torno de 3\%. O desempenho brasileiro também ficou abaixo da performance de outros países emergentes como a Coréia do Sul, China, México e Chile. A continuar nesta velocidade o Brasil deve demorar cerca de 100 anos para dobrar sua renda per capita, e nem assim vai chegar próximo do padrão de vida que hoje os países desenvolvidos desfrutam. Dessa forma, podemos afirmar que a economia brasileira se encontra numa situação de semiestagnação.

Nesse contexto, a pergunta relevante a ser feita é: o que fazer para acelerar, de forma sustentável, o ritmo de crescimento da economia brasileira? Existem duas respostas para essa pergunta. A primeira, baseada nos modelos de crescimento de inspiração neoclássica e na metodologia da contabilidade do crescimento, estabelece que a razão para o pífio crescimento da economia brasileira nos últimos 25 anos deve ser buscada no lado da oferta da economia. Mais especificamente, os problemas da economia brasileira seriam uma baixa taxa de poupança doméstica - devido à poupança negativa do setor público e ao baixo incentivo a poupança do setor privado em função da elevada carga tributária e do sistema de repartição vigente na previdência social brasileira - e um baixo dinamismo tecnológico expresso numa reduzida taxa de crescimento da produtividade total dos fatores de produção. Nesse contexto, a retomada do crescimento da economia brasileira a taxas mais expressivas exigiria uma reforma previdenciária que atuasse no sentido de aumentar a poupança do setor público e uma nova rodada de abertura comercial da economia brasileira com o intuito de estimular o crescimento da produtividade do trabalho nas empresas brasileiras.

A segunda resposta se baseia na ideia de que o modelo macroeconômico adotado pelo Brasil na última década atua no sentido de contrair a expansão da demanda agregada e, portanto, de impedir o crescimento do produto real. Isso porque a combinação de elevadas taxas reais de juros com a política de geração de superávits primários crescentes teria o efeito de deprimir a demanda agregada e, portanto, o crescimento do PIB. Nesse contexto, a solução para o problema de semiestagnação existente na economia brasileira seria o abandono puro e simples do modelo macroeconômico vigente, baseado no tripé metas de inflação-câmbio flutuante-superávit primário.

Do ponto de vista adotado no presente artigo, as duas posições acima mencionadas são equivocadas. Como ficará claro ao longo das seções seguintes, acreditamos que os fatores que limitam o crescimento econômico no longoprazo devem ser buscados no lado da demanda, não no lado da oferta da economia. No entanto, rejeitamos a visão keynesiana ingênua de que o crescimento pode ser estimulado por intermédio de qualquer política que aumente a demanda agregada. A crise fiscal do Estado Brasileiro impõe limites claros e estreitos para uma política de indução do crescimento econômico por intermédio da expansão dos gastos de consumo corrente do governo. 
Isso posto, o presente artigo está estruturado em cinco seções, incluindo a presente introdução. $\mathrm{Na}$ segunda seção apresentaremos um breve retrospecto do crescimento da economia brasileira nas últimas duas décadas. A terceira seção apresenta a teoria do crescimento puxado pela demanda agregada, de acordo com a qual a taxa de crescimento de longo prazo do produto real é uma média ponderada da taxa de crescimento dos gastos do governo em consumo corrente e da taxa de crescimento das exportações. Na quarta seção, com base na metodologia desenvolvida por Atesoglu (2002), realizamos alguns testes econométricos para a hipótese de que o crescimento da economia brasileira é puxado pela demanda agregada. Os resultados dos testes econométricos mostram que $95 \%$ do crescimento do PIB real no período 1991-2005 é explicado por variáveis do lado da demanda agregada da economia. Além disso, com base na metodologia desenvolvida por Ledesma e Thirlwall (2002), mostramos que a taxa natural de crescimento da economia brasileira é endógena, aumentando significativamente nos períodos de boom. Dessa forma, não parecem existir restrições do lado da oferta da economia para um crescimento mais acelerado da economia brasileira. Por fim, a quinta seção sumariza as conclusões obtidas ao longo deste artigo.

\section{O CRESCIMENTO DA ECONOMIA BRASILEIRA NAS ÚLTIMAS DUAS DÉCADAS}

Nas últimas duas décadas a economia brasileira vem se defrontando com uma forte desaceleração do ritmo de crescimento do produto interno bruto. Com efeito, a taxa média de crescimento da economia brasileira foi superior a $7 \%$ ao ano no período 1950-1980. Na década de 1980, esse crescimento sofre uma brutal desaceleração. No período 1981-1990, a assim chamada década perdida, a taxa média de crescimento foi inferior a $2 \%$ ao ano. No período compreendido entre $1991 \mathrm{e}$ 2000, a taxa média de crescimento do PIB se acelera para cerca de 2,7\% ao ano, ficando ainda muito abaixo da média do período 1950-1980. Por fim, no período 2001-2005, a taxa média de crescimento do PIB volta a cair, situando-se em cerca de $2,2 \%$ ao ano.

Nesse contexto, a pergunta fundamental a ser feita é: o que aconteceu com a economia brasileira para que a taxa de crescimento tenha se reduzido de forma tão dramática com respeito a sua média histórica? Com efeito, na década de 1980 se creditava a desaceleração do crescimento da economia brasileira aos efeitos acumulados da crise da dívida externa e a quase hiperinflação experimentada ao longo da década. Contudo, durante os anos 1990, a dívida externa foi equacionada com o "Plano Brady" e a inflação foi debelada com o Plano Real e, mesmo assim, a economia brasileira não voltou a crescer ao mesmo ritmo experimentado durante o período 1950-1980.

A explicação tradicional para a desaceleração do crescimento da economia brasileira se baseia nos exercícios de growth accounting. Segundo essa metodologia, o crescimento da economia brasileira se desacelerou devido à redução da taxa de 
crescimento da oferta de fatores de produção — principalmente de capital - e a redução do ritmo de progresso tecnológico da economia brasileira pós-segundo choque do petróleo (Barbosa, 2006). Essa desaceleração é devida a uma série de ineficiências herdadas do assim chamado modelo de substituição de importações prevalecente no Brasil até o final da década de 1970 (Franco, 1999). Ainda segundo essa metodologia, a economia brasileira poderia, nas condições atuais, crescer a uma taxa não superior a 3,5\% a.a., caso se queira manter o compromisso com a estabilidade da taxa de inflação.

Uma explicação alternativa para a desaceleração do crescimento tem seu foco nas condições de demanda, ao invés das condições de oferta da economia. Segundo essa linha de interpretação, o crescimento de longo prazo é puxado pela demanda agregada, de tal maneira que é de fundamental importância a análise dos fatores de impulsionam o crescimento da demanda agregada. Nesse contexto, o modelo de crescimento da economia brasileira até o final da década de 1970 esteve baseado na expansão dos gastos de consumo em bens de consumo duráveis de luxo, expansão essa viabilizada por uma política ativa de concentração de renda (Bresser-Pereira, 2004, p. 181). ${ }^{1}$

Esse modelo de crescimento se esgotou na década de 1980 em função da redemocratização do país. Com efeito, a redemocratização colocou como primeiro ponto na agenda política do Brasil a redução das enormes desigualdades na distribuição de renda. Dessa forma, não seria mais possível puxar o crescimento da demanda agregada por intermédio de aumentos do consumo de bens duráveis viabilizados por aumentos no grau de concentração de renda. O problema da economia brasileira, segundo essa linha de interpretação, é que não se colocou no lugar uma fonte alternativa de expansão da demanda agregada que viabilizasse a manutenção do crescimento da economia brasileira a taxas elevadas.

\section{CRESCIMENTO PUXADO PELA DEMANDA AGREGADA: A VISÃO KEYNESIANA}

\section{Endogenidade de longo prazo da disponibilidade dos "fatores de produção"}

Os modelos de crescimento neoclássicos supõem que o limite fundamental ao crescimento de longo prazo é a disponibilidade de fatores de produção. A demanda agregada é relevante apenas para explicar o grau de utilização da capacidade produtiva, mas não tem nenhum impacto direto na determinação do ritmo de expansão da capacidade produtiva. No longo prazo vale a "Lei de Say", ou seja, a oferta (disponibilidade de fatores de produção) determina a demanda agregada.

Mas será verdade que a disponibilidade de fatores de produção é independen-

\footnotetext{
${ }^{1}$ Sobre a relação entre concentração de renda e crescimento da demanda por bens de consumo duráveis, ver Furtado (1986, p. 208).
} 
te da demanda? Essa questão foi inicialmente analisada por Kaldor (1988), dando origem a assim chamada teoria do crescimento puxado pela demanda agregada. A premissa básica dos modelos de crescimento puxados pela demanda agregada é que os meios de produção utilizados numa economia capitalista moderna são eles próprios bens que são produzidos dentro do sistema. Dessa forma, a "disponibilidade" de meios de produção nunca pode ser considerada como um dado independente da demanda pelos mesmos. Nesse contexto, o problema econômico fundamental não é a alocação de um dado volume de recursos entre uma série de alternativas disponíveis, mas sim a determinação do ritmo no qual esses recursos são criados (Setterfield, 1997, p. 50).

Para que possamos compreender a endogenidade de longo prazo da disponibilidade de fatores de produção, comecemos inicialmente analisando a disponibilidade de capital. A quantidade existente de capital num dado ponto do tempo - ou melhor, a capacidade produtiva existente na economia - é resultante das decisões passadas de investimento em capital fixo. Daqui se segue que o estoque de capital não é uma constante determinada pela "natureza", mas depende do ritmo no qual os empresários desejam expandir o estoque de capital existente na economia.

Dessa forma, o condicionante fundamental do "estoque de capital" é a decisão de investimento. O investimento, por sua vez, depende de dois conjuntos de fatores: i) o custo de oportunidade do capital (largamente influenciado pela taxa básica de juros controlada pelo Banco Central); ii) as expectativas a respeito do crescimento futuro da demanda por bens e serviços. Nesse contexto, se os empresários anteciparem um crescimento firme da demanda pelos bens e serviços produzidos pelas suas empresas - como é de esperar no caso de uma economia que esteja apresentando um crescimento forte e sustentável ao longo do tempo - então eles irão realizar grandes investimentos na ampliação da capacidade de produção.

Em outras palavras, o investimento se ajusta ao crescimento esperado da demanda, desde que seja atendida uma restrição fundamental, a saber: a taxa esperada de retorno do capital seja maior do que o custo do capital. Sendo assim, atendida a condição acima referida, a "disponibilidade de capital" não pode ser vista como um entrave ao crescimento de longo prazo.

É verdade que no curto e médio-prazo a produção não pode aumentar além do permitido pela capacidade física de produção da economia. No longo prazo, contudo, a capacidade de produção pode ser ampliada - por intermédio do investimento em capital físico - de forma a atender a demanda agregada por bens e serviços (Kaldor, 1988, p. 157).

Uma objeção trivial a essa argumentação é que o investimento depende para a sua realização de "poupança prévia", ou seja, qualquer aumento dos gastos de investimento requer que, previamente à sua realização, haja um aumento da taxa de poupança da economia. Nesse contexto, argumentariam os economistas neoclássicos, a "disponibilidade de capital" se acha limitada pela fração da renda que uma determinada sociedade está disposta a não consumir. A poupança assim defi- 
nida é determinada pela poupança privada (famílias + empresas), pela poupança do governo e pela poupança externa.

A relação entre poupança e investimento objeto de intenso debate entre os economistas (Neo-)Clássicos e Keynesianos após a publicação da Teoria Geral do Emprego, do Juro e da Moeda de John Maynard Keynes. ${ }^{2}$ Segundo Keynes não é verdade que o investimento necessite de poupança prévia. Com efeito, a realização dos gastos de investimento exige tão somente a criação de liquidez por parte do sistema financeiro.Trata-se da assim chamada demanda por moeda devido ao motivo finance (Carvalho, 1992, p. 148-153). Se os bancos estiverem dispostos a estender as suas linhas de crédito - ainda que de curta maturidade — em condições favoráveis, então será possível que as empresas iniciem a implementação dos seus projetos de investimento, encomendando máquinas e equipamentos junto aos produtores de bens de capital. Uma vez realizado o gasto de investimento, será criada uma renda agregada de tal magnitude que, ao final do processo, a poupança agregada irá se ajustar ao novo valor do investimento em capital físico. A poupança assim criada poderá então ser utilizada para o funding das dívidas de curto-prazo das empresas junto aos bancos comerciais, ou seja, as empresas poderão - por intermédio de lucros retidos, venda de ações ou colocação de títulos no mercado - "liquidar" as dívidas contraídas junto aos bancos comerciais no momento em que precisavam de liquidez para implementar os seus projetos de investimento. A poupança se ajusta sempre, e de alguma maneira, ao nível de investimento desejado pelos empresários (Davidson, 1986). ${ }^{3}$

Os entraves a expansão da capacidade produtiva são de natureza financeira, mais especificamente, referem-se ao custo de oportunidade do capital. As empresas estarão dispostas a ajustar o tamanho de sua capacidade produtiva ao crescimento previsto da demanda desde que a taxa esperada de retorno dos novos projetos de investimento seja superior ao custo de oportunidade do capital. Grosso modo, podemos definir o custo do capital como sendo igual a taxa média de juros que a empresa tem que pagar pelos fundos requeridos pelo financiamento dos seus projetos de investimento. Existem três fontes de fundos para o financiamento dos projetos de investimento, a saber: lucros retidos, endividamento e emissão de ações. Dessa forma, o custo do capital é uma média do custo de cada uma dessas fontes de financiamento ponderada pela sua participação no passivo total da empresa.

\footnotetext{
${ }^{2}$ Sobre o debate entre Keynes e os Clássicos sobre a relação entre poupança e investimento e a determinação da taxa de juros ver Oreiro (2000).

${ }^{3}$ Deve-se observar que a determinação da poupança pelo investimento ocorre também numa economia que opera em condições de "pleno emprego". Com efeito, tal como argumentado por Kaldor (1956), numa economia que opera ao longo de uma trajetória de crescimento balanceado com pleno emprego da força de trabalho, um aumento da taxa de investimento irá resultar num aumento das margens de lucro, ocasionando assim uma redistribuição de renda dos trabalhadores para os capitalistas. Como a propensão a poupar dos capitalistas é superior a propensão a poupar dos trabalhadores, esse aumento da participação dos lucros na renda irá resultar num aumento da taxa agregada de poupança.
} 
O que dizer sobre a disponibilidade de trabalho? Será que a quantidade de trabalho pode ser vista como um obstáculo ao crescimento da produção no longo prazo? Dificilmente a disponibilidade de trabalhadores pode ser vista como um obstáculo ao crescimento. Isso por uma série de razões. Em primeiro lugar, o número de horas trabalhadas, dentro de certos limites, pode aumentar rapidamente como resposta a um aumento do nível de produção.

Em segundo lugar, a taxa de participação — definida como o percentual da população economicamente ativa que faz parte da força de trabalho - pode aumentar como resposta a um forte acréscimo da demanda de trabalho (Thirlwall, 2002, p. 86). Com efeito, nos períodos nos quais a economia cresce rapidamente, o custo de oportunidade do lazer - medido pela renda "perdida" pelo indivíduo que "escolhe" não trabalhar (jovens, mulheres casadas e aposentados) — tende a ser muito elevado, induzindo um forte crescimento da taxa de participação. Nesse contexto, a taxa de crescimento da força de trabalho pode se acelerar em virtude do ingresso de indivíduos que, nos períodos anteriores, haviam decidido permanecer fora da força de trabalho.

Por fim, devemos ressaltar que a população e a força de trabalho não são um dado do ponto de vista da economia nacional. Isso porque uma eventual escassez de força de trabalho - mesmo que seja de força de trabalho qualificada — pode ser sanada por intermédio da imigração de trabalhadores de países estrangeiros. Por exemplo, países como a Alemanha e a França puderam sustentar elevadas taxas de crescimento durante os anos 1950 e 1960 com a imigração de trabalhadores da periferia da Europa (Espanha, Portugal, Grécia, Turquia e Sul da Itália).

O último elemento a ser considerado é o progresso tecnológico. Será que o ritmo de "inovatividade" da economia pode ser considerado como uma restrição ao crescimento de longo prazo? Se considerarmos o progresso tecnológico como exógeno, então certamente o crescimento será limitado pelo ritmo na qual a tecnologia é expandida. Contudo, o progresso tecnológico não é exógeno ao sistema econômico. Em primeiro lugar, o ritmo de introdução de inovações por parte das empresas é, em larga medida, determinado pelo ritmo de acumulação de capital; haja vista que a maior parte das inovações tecnológicas é "incorporada" nas máquinas e equipamentos recentemente produzidos. ${ }^{4}$ Dessa forma, uma aceleração da taxa de acumulação de capital — induzida, por exemplo, por uma perspectiva mais favorável de crescimento da demanda - induz um maior ritmo de progresso tecnológico e, portanto, de crescimento da produtividade do trabalho.

\footnotetext{
${ }^{4}$ Essa ideia foi pioneiramente apresentada por Kaldor (1957) por intermédio da sua "função de progresso técnico", a qual estabelece a existência de uma relação estrutural entre a taxa de crescimento do produto por trabalhador e a taxa de crescimento do capital por trabalhador. Segundo Kaldor não é possível separar o crescimento da produtividade que advém da incorporação de novas tecnologias daquela parte que resulta de um aumento do capital por trabalhador, uma vez que a maior parte das inovações tecnológicas que aumentam a produtividade do trabalho exigem o emprego de um volume maior de capital por trabalhador por se acharem incorporadas em novas máquinas e equipamentos.
} 
Em segundo lugar, aquela parcela "desincorporada" do progresso tecnológico é causada por "economias dinâmicas de escala" como o "learning-by-doing". Dessa forma, se estabelece uma relação estrutural entre a taxa de crescimento da produtividade do trabalho e a taxa de crescimento da produção, a qual é conhecida na literatura econômica como "lei de Kaldor-Verdoon" (Ledesma, 2002). Nesse contexto, um aumento da demanda agregada, ao induzir uma aceleração da taxa de crescimento da produção, acaba por acelerar o ritmo de crescimento da produtividade do trabalho.

Como corolário de toda essa argumentação, segue-se que o conceito de "produto potencial" ou "nível de produção de pleno emprego", tão caro as abordagens neoclássicas de crescimento econômico, é essencialmente um conceito de curto-prazo, o qual ignora o fato de que a disponibilidade de fatores de produção e o próprio ritmo do progresso tecnológico são variáveis endógenas no processo de crescimento e desenvolvimento econômico.

\section{Determinantes de longo prazo do crescimento econômico}

Se a disponibilidade de fatores de produção não pode ser vista como o determinante do crescimento econômico no longo prazo, então quais são os fatores que determinam o crescimento? No longo prazo o determinante último da produção é a demanda agregada. Se houver demanda, as firmas irão responder por intermédio de um aumento da produção e da capacidade produtiva, desde que sejam respeitadas duas condições: i) a margem de lucro seja suficientemente alta para proporcionar aos empresários a taxa desejada de retorno sobre o capital; ii) a taxa realizada de lucro seja maior do que o custo do capital. Nessas condições, a taxa de crescimento do produto real será determinada pela taxa de crescimento da demanda agregada autônoma, ou seja, pelo crescimento daquela parcela da demanda agregada que é, em larga medida, independente do nível e/ou da variação da renda e da produção agregada.

Em economias abertas, os componentes autônomos da demanda agregada são dois, a saber: as exportações e os gastos do governo. ${ }^{5}$ Os gastos com investimento não são um componente autônomo da demanda agregada, uma vez que a decisão de investimento em capital fixo é fundamentalmente determinada pelas expectativas empresariais a respeito da expansão futura do nível de produção e de vendas em consonância com a assim chamada hipótese do acelerador do investimento

\footnotetext{
${ }^{5}$ Deve-se fazer aqui uma distinção importante entre os gastos de consumo corrente do governo e os gastos de investimento. Embora ambos os tipos de dispêndio governamental sejam "autônomos" com respeito ao nível e/ou à variação da renda corrente, os gastos de investimento do governo geram uma externalidade positiva sobre o investimento privado, razão pela qual uma política de crescimento baseada na expansão fiscal deverá privilegiar o aumento dos gastos de investimento, ao invés do aumento dos gastos de consumo corrente. Sobre os efeitos do investimento público sobre o crescimento de longo prazo ver, Oreiro, Silva e Fortunato (2008).
} 
(Harrod, 1939). Em outras palavras, o investimento não é uma variável "exógena" do ponto de vista do processo de crescimento, uma vez que o mesmo é induzido pelo crescimento do nível de renda e produção. ${ }^{6}$ Sendo assim, o crescimento de longo prazo do nível de renda e produção será uma média ponderada entre a taxa de crescimento das exportações e a taxa de crescimento dos gastos do governo.

Para uma pequena economia aberta que não dispõe de uma moeda aceita como reserva de valor internacional, a taxa de crescimento das exportações é a variável exógena por excelência. Isso porque se a taxa de crescimento dos gastos do governo for maior do que a taxa de crescimento das exportações, então o produto e a renda doméstica irão crescer mais do que as exportações. Se a elasticidade-renda das importações for maior do que um (como é usual em economias abertas), então as importações irão crescer mais do que as exportações, gerando um déficit comercial crescente e, provavelmente, insustentável no longo prazo.

A taxa de crescimento das exportações é igual ao produto entre a elasticidade-renda das exportações $(\varepsilon)$ e a taxa de crescimento da renda do resto do mundo (z). ${ }^{7}$ Isso posto, podemos concluir que a taxa potencial de crescimento do produto real $\left(\mathrm{g}^{*}\right)$, a partir da abordagem Keynesiana do crescimento puxado pelo demanda agregada, é dada por:

$$
g^{*}=\varepsilon \mathrm{Z}
$$

\section{O CRESCIMENTO DA ECONOMIA BRASILEIRA É PUXADO PELA DEMANDA AGREGADA? ALGUNS TESTES EMPÍRICOS}

Ao longo desta seção fazermos alguns testes econométricos sobre a hipótese de crescimento puxado pela demanda agregada para a economia brasileira. Inicialmente, mostraremos que determinadas variáveis do lado da demanda agregada tem um papel fundamental na explicação do crescimento da economia brasileira no período $1991-2005 .{ }^{8} \mathrm{Em}$ particular, mostraremos que as exportações e os gastos de consumo corrente do governo são variáveis exógenas no crescimento de longo prazo, corroborando assim a teoria do crescimento puxado pela demanda agregada apresentada na terceira seção. Na sequência mostraremos que a taxa natural de crescimento da economia brasileira é endógena de forma que as condições de oferta da economia não impõem uma restrição efetiva (binding) ao crescimento da economia brasileira. Nossas estimativas com base nos dados trimestrais de crescimento e desemprego da economia brasileira no período

\footnotetext{
${ }^{6} \mathrm{Na}$ quarta seção demonstraremos a validade empírica dessa hipótese para a economia brasileira.

${ }^{7}$ Supondo que os termos de troca permaneçam constantes ao longo do tempo.

${ }^{8}$ O IBGE refez os cálculos do PIB entre 1995-2006. Como a série analisada é trimestral e o período de análise do presente estudo vai de 1991 a 2005, os dados utilizados nas estimativas são aqueles coletados com a antiga metodologia do IBGE.
} 
1980-2002 mostram que a taxa natural de crescimento pode variar de 5,2\% a.a. à $8 \%$ a.a. nos períodos de boom.

\section{Testando a hipótese de crescimento puxado pela demanda agregada}

$\mathrm{Na}$ presente sub-seção utilizaremos a metodologia desenvolvida por Atesoglu (2002) para testar a hipótese de crescimento puxado pela demanda agregada para a economia brasileira. Para tanto, analisaremos a relação entre o PIB real (Y) e o nível de exportações real (X), o nível real de investimentos ${ }^{9}$ (I), o nível real de consumo do governo (G) e a oferta de moeda real (M2 deflacionada).

A origem dos dados para PIB real, exportações reais, investimentos reais, e consumo real do governo são do Sistema de Contas Nacionais fornecidos pelo Instituto Brasileiro de Geografia e Estatística (IBGE/SCN). A série da oferta de moeda tem como fonte o Banco Central do Brasil (BCB). Todas as séries são deflacionadas pelo Índice Geral de Preços (IGP-DI) calculado pela Fundação Getulio Vargas (FGV). Todas as variáveis foram transformadas de tal forma que seus valores em 1991 são iguais a $100(1991=100)$ e sobre estes índices foram aplicados logaritmos naturais. ${ }^{10}$ Como consequência, os coeficientes estimados fornecem as elasticidades entre as variáveis. O período de estudo vai do primeiro trimestre de 1991 ao último trimestre de 2005, abrangendo 60 trimestres.

Para verificar a possibilidade de existência de tendência estocástica nas variáveis, foram utilizados os testes raiz unitária: Dickey-Fuller Aumentado (ADF, teste $\mathrm{t})$, Phillips-Perron (PP, teste z) e Dickey-Fuller ajustado à tendência (DF-GLS), além do teste de estacionariedade KPSS, proposto por Kwiatkowski, Phillips, Schmidt e Shin (1992). ${ }^{11}$ A decisão quanto à inclusão ou não da constante e/ou da tendência, além do número de defasagens para cada série, foi definido de acordo com o critério de Schwarz (SC) e de Newey-West (NW), da significância estatística dos parâmetros estimados, e dos testes de diagnósticos usuais, partindo sempre da dinâmica geral para a particular (lag inicial $=10$ ). Os resultados são apresentados na Tabela 1. Eles indicam que todas as variáveis são integradas de ordem um, ou I(1), sendo, portanto, não estacionárias.

\footnotetext{
${ }^{9}$ Público e privado.

${ }^{10}$ A denominação $\mathrm{L}$ antes de cada variável indica sua forma logarítmica enquanto DL indica a primeira diferença dos logaritmos.

${ }^{11}$ Seguindo as sugestões de Maddala (2001), empregamos a análise confirmatória e adotamos o nível de significância de $10 \%$ e, em caso de contradição nos resultados, demos preferência aos testes de raiz unitária.
} 


\begin{tabular}{|c|c|c|c|c|c|c|c|c|}
\hline \multirow[b]{2}{*}{ Variável } & \multicolumn{4}{|c|}{ ADF } & \multicolumn{4}{|c|}{ PP } \\
\hline & Def. & $\begin{array}{l}\text { Termos } \\
\text { Determ. }\end{array}$ & Teste & $\begin{array}{c}\text { Valor } \\
\text { Crítico } \\
10 \%\end{array}$ & Def. & $\begin{array}{l}\text { Termos } \\
\text { Determ. }\end{array}$ & Teste & $\begin{array}{c}\text { Valor } \\
\text { Crítico } \\
10 \%\end{array}$ \\
\hline LY & 1 & $\mathrm{~N}$ & 0,70 & $-1,61$ & 2 & CT & $-3,15$ & $-3,17$ \\
\hline$D(L Y)$ & 0 & $\mathrm{~N}$ & $-10,78$ & $-1,61$ & 11 & $\mathrm{~N}$ & $-11,76$ & $-1,61$ \\
\hline$L X$ & 2 & $N$ & 1,64 & $-1,61$ & 18 & $\mathrm{~N}$ & 1,56 & $-1,61$ \\
\hline$D(L X)$ & 1 & N & $-9,52$ & $-1,61$ & 21 & $\mathrm{~N}$ & $-8,45$ & $-1,61$ \\
\hline LI & 0 & $\mathrm{~N}$ & 0,59 & $-1,61$ & 57 & $\mathrm{~N}$ & 1,25 & $-1,61$ \\
\hline$D(L I)$ & 0 & $\mathrm{~N}$ & $-7,77$ & $-1,61$ & 57 & $\mathrm{~N}$ & $-12,88$ & $-1,61$ \\
\hline LG & 4 & $\mathrm{~N}$ & 0,82 & $-1,61$ & 15 & $\mathrm{~N}$ & 1,41 & $-1,61$ \\
\hline $\mathrm{D}(\mathrm{LG})$ & 3 & $\mathrm{~N}$ & $-3,21$ & $-1,61$ & 28 & $\mathrm{~N}$ & $-15,62$ & $-1,61$ \\
\hline LM2 & 0 & $\mathrm{CT}$ & $-2,31$ & $-3,17$ & 0 & $\mathrm{CT}$ & $-2,31$ & $-3,17$ \\
\hline $\mathrm{D}(\mathrm{LM} 2)$ & 1 & N & $-3,02$ & $-1,61$ & 2 & $\mathrm{~N}$ & $-6,42$ & $-1,61$ \\
\hline
\end{tabular}

\begin{tabular}{|c|c|c|c|c|c|c|c|c|}
\hline \multirow[b]{2}{*}{ Variável } & \multicolumn{4}{|c|}{ DF-GLS } & \multicolumn{4}{|c|}{ KPSS } \\
\hline & Def. & $\begin{array}{l}\text { Termos } \\
\text { Determ. }\end{array}$ & Teste & $\begin{array}{c}\text { Valor } \\
\text { Crítico } \\
10 \%\end{array}$ & Def. & $\begin{array}{l}\text { Termos } \\
\text { Determ. }\end{array}$ & Teste & $\begin{array}{c}\text { Valor } \\
\text { Crítico } \\
10 \%\end{array}$ \\
\hline LY & 1 & C & $-0,59$ & $-1,61$ & 5 & CT & 0,20 & 0,12 \\
\hline$D(L Y)$ & 0 & $\mathrm{CT}$ & $-7,74$ & $-2,87$ & 48 & C & 0,39 & 0,35 \\
\hline$L X$ & 2 & $\mathrm{CT}$ & $-1,46$ & $-2,87$ & 5 & CT & 0,23 & 0,12 \\
\hline$D(L X)$ & 1 & $\mathrm{CT}$ & $-8,60$ & $-2,87$ & 18 & C & 0,15 & 0,35 \\
\hline $\mathrm{LI}$ & 0 & $\mathrm{CT}$ & $-2,44$ & $-2,86$ & 5 & CT & 0,22 & 0,12 \\
\hline $\mathrm{D}(\mathrm{LI})$ & 0 & CT & $-5,62$ & $-2,87$ & 58 & CT & 0,50 & 0,12 \\
\hline LG & 4 & CT & $-1,78$ & $-2,88$ & 5 & CT & 0,21 & 0,12 \\
\hline $\mathrm{D}(\mathrm{LG})$ & 3 & CT & $-2,21$ & $-2,88$ & 15 & C & 0,14 & 0,35 \\
\hline LM2 & 0 & CT & $-1,14$ & $-2,86$ & 6 & CT & 0,23 & 0,12 \\
\hline $\mathrm{D}(\mathrm{LM} 2)$ & 1 & C & $-2,61$ & $-1,61$ & 3 & C & 0,42 & 0,35 \\
\hline
\end{tabular}

Notas: $\mathrm{N}=$ Nenhum; $\mathrm{C}=$ Constante e; $\mathrm{CT}$ = Constante e Tendência Linear. No teste de ADF e DF-GLS o número de defasagens inicial para cada série é definido de acordo com o critério de Schwarz. Para os testes PP e KPSS aplica-se a seleção por Newey-West.

Pelo fato de que todas as séries são I(1), não há problemas de espuriedade nos resultados quando se estima a regressão com as variáveis em primeira diferença. Os resultados estão apresentados abaixo: 


\begin{tabular}{cccccc}
\hline \multicolumn{2}{l}{$\begin{array}{c}\text { Variável Dependente: DLY } \\
\text { Método: MOO }\end{array}$} & \multicolumn{7}{c}{ Observações: 59} \\
\hline Variável & C & DLX & DLI & DLG & DLM2 \\
\hline Coeficiente & $-0,0054$ & 0,1753 & 0,3228 & 0,2087 & 0,3312 \\
Erro-Padrão & 0,0076 & 0,0579 & 0,1151 & 0,0556 & 0,1537 \\
Estatística t & $-0,7094$ & 3,0296 & 2,8038 & 3,7564 & 2,1547 \\
Fator Inf. Variância & & 1,0270 & 1,0060 & 1,1810 & 1,1690 \\
\hline R-quadrado & 0,4658 & Durbin-Watson & & 2,3652 \\
R-quad. Ajustado & 0,4262 & Breusch-Godfrey & Lags: 2 & 3,2573 \\
Erro-padrão (eq.) & 0,0547 & & Lags: 4 & 3,2877 \\
Log-probabilidade & 90,3107 & Teste ARCH & Lags: 1 & 2,3725 \\
Critério de Akaike & $-2,9618$ & & Lags: 2 & 3,4820 \\
Critério de Schwarz & $-2,7158$ & & Lags: 4 & 4,0796 \\
Estatística F & 11,7699 & Teste de White & & 15,4668 \\
Teste de Chow & 3,0986 & Teste de Breusch-Pagan & 4,3934 \\
Teste Ramsey RESET & 1,8084 & Teste de Doornik-Hansen & 4,7683 \\
\hline
\end{tabular}

Todas as variáveis não determinísticas têm os sinais esperados e são significativas ao nível de $5 \%$ ou $1 \%$, inclusive conjuntamente (Estatística F). Os testes de diagnósticos sobre especificação do modelo (Ramsey RESET), presença de mudança estrutural (Chow), existência de multicolinearidade (Fator de Inflação da Variância), além dos tradicionais critérios de seleção (Akaike - AIC e Schwarz - SIC) validam a parametrização escolhida. Testes sobre os resíduos para verificar a existência de problemas de autocorrelação (Durbin-Watson e Breusch-Godfrey), de heterocedasticidade (White, Breusch-Pagan e ARCH), e de normalidade (Doornik-Hansen) não mostraram evidências da presença dos problemas em consideração.

As variáveis do lado direito da equação de regressão explicam cerca $47 \%$ da variação do PIB. A oferta de moeda tem o maior impacto sobre PIB. Um aumento de $1 \%$ na primeira eleva o PIB em $0,33 \%$.

A análise de dinâmica de curto prazo realizada na Tabela $2-$ feita com a supressão das tendências nas variáveis via diferenciação - descarta informações importantes sobre o longo prazo. Dado que as variáveis envolvidas são todas I(1), existe a possibilidade de cointegração. Destarte, com base na teoria elaborada em Granger (1981) e Engle e Granger (1987) vamos integrar dinâmicas de curto prazo com equilíbrios de longo prazo.

A regressão estática das variáveis em nível (Tabela 3) faz parte do procedimento Engle-Granger de dois estágios (EG) para se testar a cointegração. Caso as variáveis sejam cointegradas - o resíduo for estacionário ou $\mathrm{I}(0)$, podemos obter 
parâmetros consistentes de longo prazo e o termo de correção de erros para a regressão de curto prazo.

O modelo de longo prazo é validado pelos testes de diagnósticos efetuados. Todavia, os resíduos apresentam evidências de correlação serial. Assim, apresentamos os valores dos erros-padrão (e das estatísticas t) corrigidos pela matriz de covariância consistente a autocorrelação e a heterocedasticidade de White (HAC). As variáveis LX e LI, que eram significativas a 5\%, passam a ser significantes a $1 \%$, enquanto LG é significante a $1 \%$ e LM2 não apresenta significância, em ambos os casos (com ou sem correção). ${ }^{12}$

Alternativamente, para resolver o problema de autocorrelação residual, escolhemos estimar a regressão de longo prazo dinâmica, por um modelo autoregressivo de defasagem distribuída, ou Autoregressive Distributed Lag (ADL). ${ }^{13}$ Este modelo passa pelos testes de diagnósticos. Em termos de significância dos parâmetros, LX, LI e LG continuam significantes (a pelo menos 10\%) e LM2, não.

Tabela 3: Modelo de longo prazo (EG)

\begin{tabular}{|c|c|c|c|c|c|}
\hline \multicolumn{6}{|c|}{ Variável Dependente: LY } \\
\hline \multicolumn{2}{|l|}{ Método: MQO } & \multicolumn{4}{|c|}{ Observações: 59 (ADL) e 60 Reg. Estática } \\
\hline Variável & C & $L X$ & $\mathrm{LI}$ & LG & LM2 \\
\hline \multicolumn{6}{|c|}{ Regressão Estática } \\
\hline Coeficiente & 0,6599 & 0,0687 & 0,3172 & 0,4134 & 0,0533 \\
\hline Erro-Padrão & 0,5207 & 0,0278 & 0,1193 & 0,0680 & 0,0543 \\
\hline Estatística t & 1,2674 & 2,4735 & 2,6598 & 6,0805 & 0,9818 \\
\hline Erro-Padrão (HAC) & 0,4864 & 0,0239 & 0,0847 & 0,1067 & 0,0634 \\
\hline Estatística t (HAC) & 1,3569 & 2,8783 & 3,7428 & 3,8758 & 0,8397 \\
\hline Fator Inf. Variância & & 1,7170 & 2,3850 & 3,4210 & 4,6910 \\
\hline \multicolumn{6}{|c|}{ Autorregressivo de Defasagem Distribuída } \\
\hline Coeficiente & 1,1726 & 0,0389 & 0,0793 & 0,5966 & 0,0316 \\
\hline Teste de Wald (Est.F) & 3,7628 & 1,5588 & 3,1683 & 10,9340 & 0,1173 \\
\hline R-quadrado & 0,8743 & Durbin-Watson & & & 1,9849 \\
\hline R-quad. Ajustado & 0,8513 & Breusch-Godfrey & & Lags: 2 & 1,2114 \\
\hline Erro-padrão (eq.) & 0,0468 & & & Lags: 4 & 1,9216 \\
\hline Log-probabilidade & 102,3642 & Teste ARCH & & Lags: 1 & 0,0837 \\
\hline Critério de Akaike & $-3,1310$ & & & Lags: 2 & 0,4808 \\
\hline Critério de Schwarz & $-2,7789$ & & & Lags: 4 & 0,7408 \\
\hline Estatística F & 37,8855 & Teste de White & & & 57,9920 \\
\hline Teste de Chow & 0,2095 & Teste de Breusch & -Pagan & & 11,1197 \\
\hline Teste Ramsey RESET & 1,9947 & Teste de Doornik & -Hansen & & 14,1386 \\
\hline
\end{tabular}

Notas: As estatísticas de diagnóstico referem-se ao modelo ADL com 1 defasagem.

\footnotetext{
${ }^{12}$ Indícios de que a autocorrelação não é tão grave neste caso.

${ }^{13} \mathrm{O}$ modelo estático com as transformações de Cochrane-Orcutt e de Prais-Winsten não altera a significância dos parâmetros.
} 
Entretanto, para que as relações estimadas não sejam espúrias, é necessário que as variáveis em questão se cointegrem. $\mathrm{O}$ passo seguinte no procedimento Engle-Granger é verificar se o resíduo da relação de longo prazo é estacionário. $\mathrm{O}$ teste empregado é o ADF. No entanto, não é aconselhável o uso dos valores presentes nas tabelas tradicionais para teste de hipótese. Essas tabelas não são preparadas para valores estimados. Assim, utiliza-se a tabela ajustada para valores estimados e para o tamanho da amostra proposta em MacKinnon (1990). O valor da estatística de teste $(-4,68)$ rejeita a presença de raiz unitária com $99 \%$ de confiança estatística, evidenciando a existência de uma relação de longo prazo entre as variáveis.

Dada a existência de variáveis cointegradas, pode-se utilizar o Modelo de Correção de Erros (MCE). Esse modelo faz a ligação entre aspectos relacionados com a dinâmica de curto prazo com os de longo prazo, isto é, permite combinar as vantagens de se modelar tanto em diferenças quanto em nível.

Tabela 4: Modelo de curto prazo (EG)

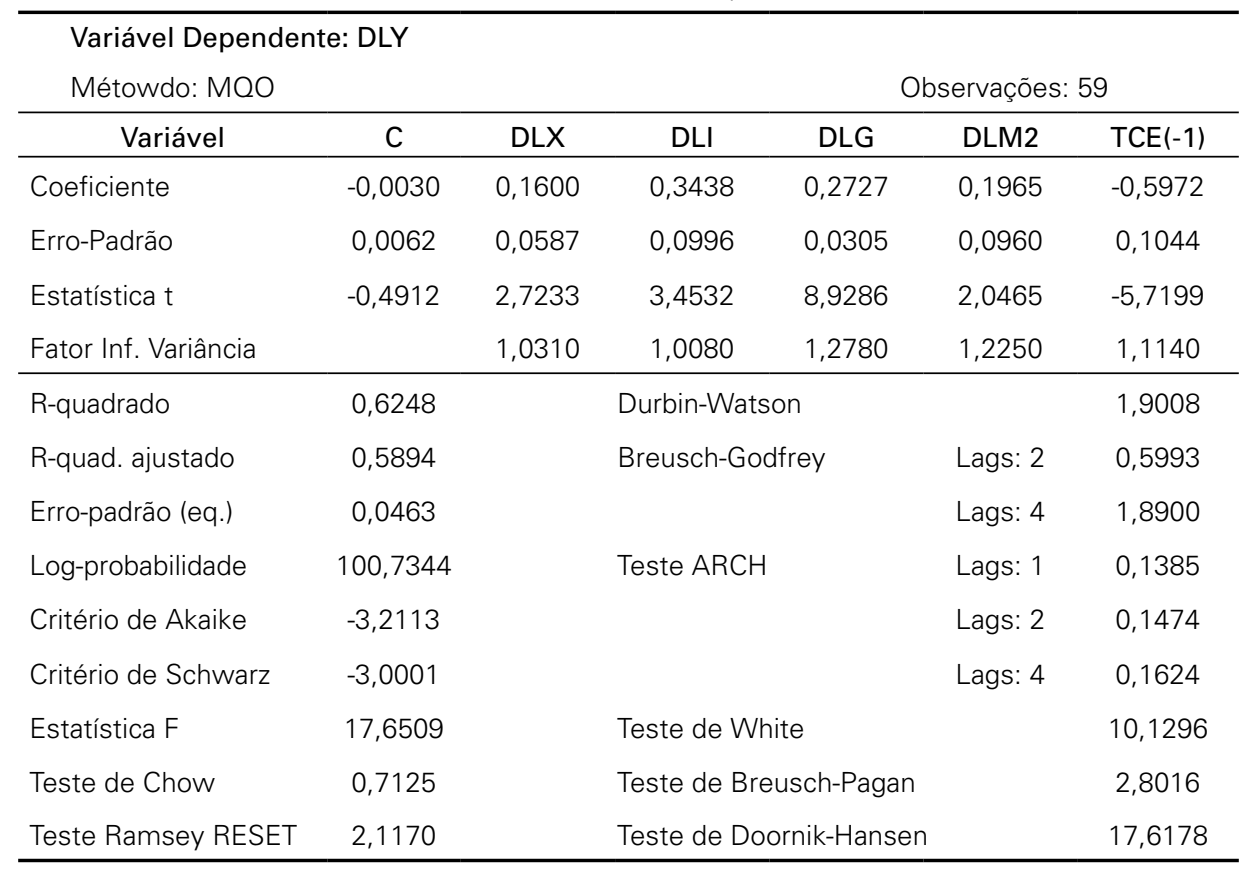

Como pode ser visto na Tabela 4, as variáveis em estudo estão diferenciadas e, portanto, são estacionárias (originalmente possuem uma raiz unitária). Para que a equação esteja balanceada, no sentido de estarem no mesmo nível de integração, é imperativo que o termo de correção de erros (TCE) seja I(0). Logo, a cointegração entre as variáveis, no sentido proposto em Engle e Granger (1987), impõe a existência de um MCE, e a recíproca é verdadeira. As elasticidades são todas significativas (a pelo menos 5\%), sendo próximas às obtidas no modelo em primeiras diferenças usual. Contudo, o coeficiente do TCE — que mensura a distância de Y e X 
em relação ao equilíbrio de longo prazo e, desse modo, informa a velocidade de ajustamento das variáveis aos ocasionais desequilíbrios - é de cerca de $60 \%{ }^{14}$

Para uma análise ainda mais robusta, utilizaremos a abordagem presente em Johansen (1988 e 1991) e Johansen e Juselius (1990) para verificar a existência de cointegração e as relações existentes para o equilíbrio de longo prazo. O procedimento de Johansen, como é conhecido, é um método de máxima verossimilhança mais geral, no qual se utiliza um sistema de equações dinâmico, ou especificamente um VAR. A abordagem sistêmica de Johansen é capaz de identificar não somente a presença de cointegração, mas também, se confirmada, o número de vetores cointegrantes existentes e a especificação destes vetores.

O número de defasagens foi definido em função dos critérios de razão de máxima verossimilhança modificada (LR), erro de predição final (FPE), Akaike (AIC), Schwarz (SIC) e Hannan-Quinn (HQ), enquanto a decisão de inclusão dos termos determinísticos baseou-se na análise visual das séries e do princípio de Pantula. Para testarmos a hipótese de cointegração e, paralelamente, caso haja, o número de vetores, utilizamos a estatística do traço e a estatística do máximo autovalor (Tabela 5).

Tabela 5: Testes de cointegração

\begin{tabular}{cccc}
\hline & \multicolumn{3}{c}{ Teste do Traço } \\
\hline Hypothesized & Autovalor & $\begin{array}{c}\text { Estatística } \\
\text { do Traço }\end{array}$ & 0,05 \\
No. of CE(s) & 0,4969 & 76,9029 & Critical Value \\
None & 0,3780 & 39,8108 & 69,8189 \\
At most 1 & 0,1523 & 14,1673 & 47,8561 \\
At most 2 & 0,0903 & 5,2455 & 29,7971 \\
At most 3 & 0,0025 & 0,1336 & 15,4947 \\
At most 4 & Teste do Máximo Autovalor & 3,8415 \\
\hline Hypothesized & & Estatística do & \\
No. of CE(s) & Autovalor & Max. Autovalor & Critical Value \\
None & 0,4969 & 37,0921 & 33,8769 \\
At most 1 & 0,3780 & 25,6435 & 27,5843 \\
At most 2 & 0,1523 & 8,9218 & 21,1316 \\
At most 3 & 0,0903 & 5,1119 & 14,2646 \\
At most 4 & 0,0025 & 0,1336 & 3,8415 \\
\hline
\end{tabular}

\footnotetext{
${ }^{14} \mathrm{O}$ sinal negativo é esperado por garantir que qualquer desequilíbrio seja compensado por meio de um movimento contrário na variável explicada.
} 
Ambos os testes sugerem a existência de um vetor de cointegração. Destarte, podemos estimar o Vetor de Correção de Erros (VEC). As elasticidades de longo prazo obtidas pelo vetor cointegrante são apresentadas na equação (2):

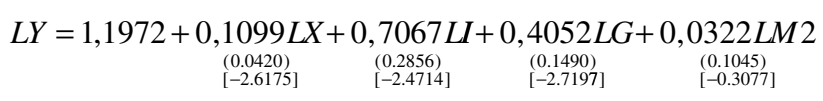

Amostra: 1992:3-2005:4; Defasagens: 1 a 5;

Assume-se uma tendência determinística nos dados.

As estimativas dos parâmetros da equação (2) são os coeficientes normalizados de cointegração, os valores em parênteses são os erros-padrão e em colchetes a estatística t. Todas as elasticidades apresentam os sinais esperados pela teoria e são estatisticamente significantes (a 5\%), com exceção, novamente, de LM2.

De forma complementar, um sumário dos resultados provindos do VEC incluindo o termo de correção de erros de cada equação e os diagnósticos básicos acerca do modelo como um todo - é apresentado na Tabela 6. Os resultados desta tabela fornecem os coeficientes de ajustamento ao equilíbrio de longo prazo, obtidos de cada uma das cinco equações múltiplas do VEC. A significância do termo de correção de erro de cada equação indica que a variável dependente ajusta-se em resposta ao desequilíbrio entre a variável dependente e as variáveis independentes, o que, por sua vez, denota a existência de endogeneidade.

Tabela 6: Sumário dos resultados do VEC

\begin{tabular}{lccccc}
\hline & $\mathrm{D}(\mathrm{LY})$ & $\mathrm{D}(\mathrm{LX})$ & $\mathrm{D}(\mathrm{LI})$ & $\mathrm{D}(\mathrm{LG})$ & $\mathrm{D}(\mathrm{LM} 2)$ \\
\hline TCE & -0.7540 & 0.9970 & 0.4946 & -0.7177 & -0.2112 \\
Erro-Padrão & $(0,2490)$ & $(0,5846)$ & $(0,2423)$ & $(0,3805)$ & $(0,2156)$ \\
Estatística t & {$[-3,0285]$} & {$[1,7053]$} & {$[2,0379]$} & {$[-1,8858]$} & {$[-0,9794]$} \\
R-quadrado Ajust. & 0.6757 & 0.4361 & 0.4121 & 0.8157 & 0.4696 \\
Erro-Padrão (eq.) & 0.0405 & 0.0952 & 0.0411 & 0.0620 & 0.0351 \\
\hline Est. LM (Autocor.) & 24.2728 & & & & \\
White (Heterosc.) & 801.1020 & & & & \\
Lutkepohl (Norm.) & 2.8940 & & & & \\
\hline
\end{tabular}

As estatísticas de teste sobre o VEC rejeitam a presença de autocorrelação, heterocedasticidade e não normalidade nos resíduos. Quanto à matriz de ajustamento, apenas os termos de correção de erro das equações do Produto e do Investimento são significativos (até 5\%). Assim, existem evidências de exogeneidade (fraca) para as variáveis de Exportação e Gastos do Governo em relação ao modelo. A oferta de moeda não se ajusta a desequilíbrios de longo prazo, todavia não é significativa no longo prazo.

A análise dos desequilíbrios de curto prazo e sua interação com a dinâmica de longo prazo fornecida pela metodologia de Engle-Granger e pelo procedimento de 
Johansen permitem algumas conclusões interessantes. Os sinais sugeridos pela teoria são observados empiricamente para o Brasil, no período em estudo. A despeito do coeficiente das elasticidades encontrados em cada modelo, podemos observar que as variáveis explicativas Exportação, Investimento e Gastos do Governo são significativas no curto e no longo prazo. A oferta de moeda é significante apenas na dinâmica de curto prazo. Desta forma, parece pouco provável que a política monetária tenha efeitos persistentes sobre o crescimento da economia brasileira. Isso porque o ritmo de crescimento do volume de meios de pagamento no sentido amplo parece não ter influência estatística sobre o comportamento do PIB real no longo prazo.

Deve-se ressaltar que, com base nos coeficientes estimados da equação de regressão 2 , para cada $1 \%$ de aumento no consumo real do governo a elevação do PIB real é de $0,40 \%$. Dessa forma, supondo que a arrecadação tributária nas três esferas de governo seja de aproximadamente $40 \%$ do PIB, um aumento dos gastos de consumo corrente do governo na ordem de $1 \%$ geraria um aumento da arrecadação tributária de aproximadamente $0,16 \%$, resultando assim num aumento de déficit público. Dada a elevada carga tributária existente na economia brasileira (cerca de $40 \%$ ) e a elevada dívida pública como proporção do PIB (cerca de $40 \%$ em termos líquidos) segue-se que, nas condições atuais, não é possível puxar de forma permanente o crescimento da economia brasileira por intermédio de um aumento dos gastos de consumo corrente do governo. A única fonte "autônoma" de demanda capaz de induzir uma aceleração do crescimento é a demanda por exportações. Em outras palavras, o modelo de crescimento da economia brasileira deve ser do tipo "export-led growth".

Exceto pela oferta de moeda, os resultados apresentados na presente seção são muito similares aos encontrados por Atesoglu (2002). As relações de causalidade dão suporte à abordagem keynesiana apresentada na seção anterior, segundo a qual as exportações e os gastos do governo são as fontes fundamentais de crescimento econômico no longo prazo, fazendo-se a ressalva de que, no caso brasileiro, em função da forte crise fiscal, não parece ser possível puxar o crescimento da economia por intermédio de uma política de expansão dos gastos de consumo corrente do governo. A saída para a retomada do crescimento da economia brasileira a altas taxas é optar por um modelo do tipo "export-led growth".

\section{A taxa natural de crescimento da economia brasileira é endógena?}

$\mathrm{Na}$ presente subseção pretendemos testar a endogeneidade da taxa natural de crescimento para a economia brasileira. Essa seção é baseada em um estudo realizado por Ledesma e Thirlwall (2002) e Thirlwall (2002). Utilizando o conceito de Okun (1962, citado por Ledesma e Thirlwall, 2002), a taxa natural de crescimento $\left(\mathrm{g}_{\mathrm{n}}\right)$ é aquela que mantém constante o nível de desemprego. Okun (1962, citado por Ledesma e Thirlwall, 2002) emprega a seguinte especificação para a variação no nível percentual do desemprego: 


$$
\Delta \% U=a-b(g)
$$

onde $\underline{\mathrm{U}}$ é o nível de desemprego, g é a taxa de crescimento do produto e $\underline{\mathrm{a}}$ e $\underline{\mathrm{b}}$ são duas constantes. Pela equação (3), quando $\Delta \% \mathrm{U}=0$, a taxa natural de crescimento é definida por $\mathrm{a} / \mathrm{b}$.

Pelo fato de que algumas pessoas deixam de procurar trabalho quando a taxa de crescimento é baixa, é possível que o coeficiente a seja subestimado. Nesse caso, a taxa de crescimento da economia também seria subestimada. Por outro lado, em períodos de elevado crescimento, parte do trabalho adicional necessário para aumentar a produção é proveniente de trabalho que estava ocioso e por horas extras. Assim, o b acaba sendo subestimado, o que leva a uma sobrestimação da taxa natural de crescimento. Assim, a taxa natural de crescimento pode ser sub ou sobrestimada dependendo de qual dos dois efeitos prevalecer.

Uma abordagem diferente para estimar a taxa natural de crescimento, na tentativa de contornar tais problemas, foi desenvolvida por Thirlwall (1979):

$$
g=a_{1}-b_{1}(\Delta \% U)
$$

$\mathrm{Na}$ equação (4), quando a variação na taxa de desemprego é zero, temos que:

$$
g=a_{1}
$$

pois $\Delta \% \mathrm{U}=0$. Assim, a taxa natural de crescimento é definida pelo intercepto da equação de regressão. O problema de se utilizar a equação (4) é que a taxa natural de crescimento é endógena e, desse modo, os coeficientes estimados serão tendenciosos.

Uma vez estimada a taxa natural de crescimento, podemos criar uma variável dummy que tome o valor 1 (um) quando a taxa de crescimento da economia for maior do que a natural estimadas pelas equações (3) ou (4) e 0 (zero), caso contrário. Com a introdução da variável dummy, temos a seguinte equação de regressão:

$$
g=a_{2}+b_{2} D+c_{2}(\Delta \% U)
$$

onde D representa a variável dummy. Na especificação da equação (6) são estimadas duas taxas naturais de crescimento. A primeira é estimada para os períodos em que a taxa de crescimento é maior do que a natural dada pela equação (4). Nesse caso, a taxa natural de crescimento é igual a $\mathrm{a}_{2}+\mathrm{b}_{2}$, considerando que $\Delta \% \mathrm{U}=0$. A segunda é estimada levando em consideração os períodos em que a taxa de crescimento é menor do que a natural dada pela equação (4). Nesse caso, a taxa natural é $\mathrm{a}_{2}$, pois $\Delta \% \mathrm{U}=0$.

Por ser uma taxa natural, seria de esperar que ela não se alterasse com variações que viessem a ocorrer na taxa de crescimento da economia. Se isso é verdade, o coeficiente da variável dummy deve ser não significativo. Caso contrário, a taxa 
natural de crescimento $\left(\mathrm{g}_{\mathrm{n}}\right)$ é endógena e responde a mudanças que venham a ocorrer na taxa de crescimento da economia $(\mathrm{g})$.

A base de dados utilizada no presente estudo para realizar a análise de regressão é composta pelas variáveis PIB e desemprego. O nível de desemprego é proveniente da Pesquisa Mensal do Emprego (PME) do Instituto Brasileiro de Geografia e Estatística (IBGE). Os dados são mensais, mas foram transformados em trimestrais a partir de uma média aritmética dos três meses de cada trimestre. ${ }^{15} \mathrm{O}$ Índice Encadeado do PIB tem como base o Sistema de Contas Nacionais do IBGE (IBGE/ SCN). ${ }^{16}$ O período de análise vai do primeiro trimestre de 1980 até o último de 2002. ${ }^{17}$ As duas variáveis foram transformadas em taxas de crescimento. Desse modo, perdeu-se a primeira observação de cada série, restando 91 observações para a realização da análise empírica.

Considerando as séries de dados trimestrais, as estimativas da taxa natural de crescimento (TNC), utilizando as equações (3) e (4), são dadas na Tabela 7:

Tabela 7: Estimação da taxa natural utilizando as equações de Okun e de Thirlwall

\begin{tabular}{l|cccccc}
\hline & método & intercepto & $\begin{array}{c}\text { coeficiente } \\
\text { angular }\end{array}$ & DW & R2 Aj. & TNC \\
\hline Equação (3) & $R R$ & $\begin{array}{c}1,61 \\
(0,99)\end{array}$ & $\begin{array}{c}-2,70^{* * *} \\
(3,49)\end{array}$ & 2,32 & 0,11 & 0,60 \\
\hline Equação (4) & MQO & $\begin{array}{c}0,59 * * * \\
(2,99)\end{array}$ & $\begin{array}{c}-0,053^{* * *} \\
(4,12)\end{array}$ & 1,89 & 0,15 & 0,59 \\
\hline
\end{tabular}

Notas: ** é significativo ao nível de $1 \%$; * é significativo ao nível de 5\%; * é significativo ao nível de 10\%. MQO é o Método dos Mínimos Quadrados Ordinários; RR é o método de regressão robusta para corrigir problemas de não normalidade dos resíduos e heterocedasticidade. DW é o valor do teste de Durbin-Watson para autocorrelação de primeira ordem; R² Aj. é o R² Ajustado; e TNC é a Taxa Natural de Crescimento.

A taxa de crescimento dada por cada uma das equações são muito semelhantes, o que dá maior credibilidade aos resultados encontrados, apesar dos possíveis problemas mencionados anteriormente.

Com uma taxa natural de crescimento em torno de $0,60 \%$ por trimestre, temos uma taxa anualizada próxima de $2,50 \%$. Assim, segundo as equações de regressão

\footnotetext{
${ }^{15}$ A partir dos dados mensais, cada ano foi dividido em quatro trimestres pela soma do desemprego de cada três meses divido por três. $1^{\circ}$ trimestre: taxa de desemprego de (Janeiro + Fevereiro + Março)/3; 2o trimestre: taxa de desemprego de (Abril + Maio + Junho)/3; 3o trimestre: taxa de desemprego de $(J u l h o+$ Agosto + Setembro)/3; 4o trimestre: taxa de desemprego de (Outubro + Novembro + Dezembro)/3.

${ }^{16}$ Série encadeada do índice trimestral de base móvel com ajuste sazonal, com média $1990=100$.

${ }^{17}$ O período de análise termina em 2002 devido a uma mudança metodológica que ocorreu na Pesquisa Mensal de Emprego a partir de 2003, inviabilizando a extensão dos testes econométricos até o período mais recente devido a mudança na base de dados.
} 
utilizadas, podemos dizer que, entre 1980 e 2002, a taxa de crescimento que mantém a taxa de desemprego constante no Brasil ficou perto de 2,50.

$\mathrm{Na}$ Tabela 8, podemos ver os resultados das equações de regressão (6). A sigla MA na Tabela 8 implica que a taxa de crescimento do PIB é uma média móvel formada por três trimestres.

Tabela 8: Estimação da taxa natural utilizando as equações de Okun e de Thirlwall com variável dummy

\begin{tabular}{l|cccccccc}
\hline & método & Intercepto & $\begin{array}{c}\text { coeficiente } \\
\text { dummy }\end{array}$ & $\begin{array}{c}\text { coeficiente } \\
\text { angular }\end{array}$ & DW & R2 Aj. & $\begin{array}{c}\text { TNC } \\
(\mathrm{g}<\mathrm{gn})\end{array}$ & $\begin{array}{c}\text { TNC } \\
\text { (g>gn) }\end{array}$ \\
\hline Equação (6) & MOO & $\begin{array}{c}-0,84^{* * *} \\
2,85^{* * *}\end{array}$ & $0,03^{* * *}$ & 2,28 & 0,61 & $-0,84$ & 2,01 \\
& & $(-4,40)$ & $(10,40)$ & $(-3,35)$ & & & & \\
\hline Equação (6) & PWER & $-0,26^{*}$ & $1,56^{* * *}$ & $0,011^{* *}$ & 1,82 & 0,54 & $-0,26$ & 1,3 \\
& & $(-1,66)$ & $(10,26)$ & $(-2,14)$ & & & & \\
\hline
\end{tabular}

Notas: ${ }^{* *}$ é significativo ao nível de $1 \% ;{ }^{*}$ é significativo ao nível de $5 \%$; ${ }^{*}$ é significativo ao nível de 10\%. MQO é o Método dos Mínimos Quadrados Ordinários; PWER é o método de Prais-Wisten para corrigir problemas de autocorrelação; PWER é o método de Prais-Wisten com erros robustos para corrigir problemas de autocorrelação e heterocedasticidade. DW é o valor do teste de Durbin-Watson para autocorrelação de primeira ordem; $R^{2}$ Aj. é o $R^{2}$ Ajustado; TNC é a Taxa Natural de Crescimento; e MA é a equação de regressão utilizando médias móveis de três trimestres.

Os resultados da equação de regressão (6) indicam que a taxa natural de crescimento responde ao crescimento que ocorre de fato na economia. Por exemplo, pelos resultados da primeira linha poderíamos dizer que em períodos da elevado crescimento, a taxa natural fica em torno de $8 \%,{ }^{18}$ enquanto em períodos de baixo crescimento ou recessão a taxa natural é negativa, ficando próxima de $-3,5 \%{ }^{19}$

Cabe lembrar que os dados são trimestrais e, desse modo, a amplitude de variação é grande. Essa é outra vantagem de se utilizar médias móveis, pois acabam suavizando as oscilações que ocorrem de um semestre para outro. Isso fica claro quando analisamos a segunda linha da Tabela 8. Nesse caso, a taxa natural de crescimento anual em períodos de bonança ficaria em torno de 5,2\%, enquanto em tempos mais tempestuosos, ela ficaria próxima de $-1 \%$.

Os testes indicam que a taxa natural de crescimento da economia brasileira é uma variável endógena, podendo ser afetada pelas condições de demanda prevalecentes na economia brasileira. Além disso, verificamos que as estimativas para a taxa natural de crescimento (nos períodos de boom) variam entre 5,2\% a.a. e $8 \%$

\footnotetext{
${ }^{18}=\left(a_{2}+b_{2}\right) \times 4=(-0,84+2,85) \times 4$. Multiplica-se por quatro porque os dados são trimestrais e estamos utilizando a taxa de crescimento natural anualizada.

${ }^{19}=\left(a_{2}\right) \times 4=(-0,84) \times 4$. Multiplica-se por quatro porque os dados são trimestrais e estamos utilizando a taxa de crescimento natural anualizada.
} 
a.a. Portanto, ao que tudo indica a economia brasileira pode crescer a taxas muito superiores a $3.5 \%$ a.a. sem gerar pressões inflacionárias.

\section{CONCLUSÕES}

Ao longo deste artigo discutimos a economia do crescimento puxado pela demanda agregada, tendo como objetivo responder a duas perguntas fundamentais, a saber: i) por que o ritmo de crescimento da economia brasileira se desacelerou nas últimas duas décadas relativamente ao período 1950-1980; ii) o que deve ser feito, em termos de formulação de política econômica, para uma aceleração sustentável do crescimento da economia brasileira?

Nossa resposta à primeira pergunta tem como fundamento a teoria do crescimento puxado pela demanda agregada. Com efeito, os testes econométricos realizados na quarta seção deste artigo mostram que $85 \%$ do crescimento do PIB real no período 1990-2005 é explicado por variáveis do lado da demanda agregada da economia, corroborando a hipótese de crescimento puxado pela demanda agregada para a economia brasileira. Além disso, com base na metodologia desenvolvida por Ledesma e Thirwall (2002), mostramos que a taxa natural de crescimento da economia brasileira é endógena, aumentando significativamente nos períodos de boom. Dessa forma, não parecem existir restrições do lado da oferta da economia para um crescimento mais acelerado da economia brasileira.

Nesse contexto, a economia brasileira teria enfrentado uma desaceleração no ritmo de crescimento no início dos anos 1980 devido ao esgotamento do padrão de expansão da demanda agregada vigente desde 1964, qual seja: a expansão dos gastos de consumo em bens duráveis de luxo viabilizada por uma crescente concentração de renda nas classes média e alta. Dessa forma, a semiestagnação da economia brasileira resulta da inexistência, nas condições atuais, de um modelo consistente de expansão da demanda agregada.

Os testes econométricos também mostraram que o multiplicador dos gastos de consumo corrente do governo é aproximadamente igual a 0,37 , de tal forma que um aumento de $1 \%$ dos gastos de consumo corrente do governo irá resultar num aumento de $0,37 \%$ do PIB real brasileiro. Tomando-se como base uma carga tributária de cerca de $40 \%$ do PIB, segue-se que um aumento de $1 \%$ dos gastos de consumo corrente do governo irá aumentar a receita tributária em apenas $0,15 \%$ do PIB. Daqui se segue que em função da crise fiscal do Estado Brasileiro, expressa pela combinação entre elevada dívida pública como proporção do PIB, carga tributária elevada e reduzido investimento público em obras de infraestrutura, não é possível puxar o crescimento da economia brasileira por intermédio de uma política de expansão dos gastos de consumo corrente do governo. 


\section{REFERÊNCIAS BIBLIOGRÁFICAS}

ASTERIOU, D. (2006). Applied Econometrics: A Modern approach using Eviews and Microfit. New York: Palgrave Macmillan.

ATESOGLU, H.S. (2002). "Growth and fluctuations in the USA: a demand oriented approach" In: SETTERFIELD, M. (org.). The Economics of Demand-Led Growth. Aldershot: Edward Elgar.

BARBOSA, F.H. (2006). "Brasil X Coreia do Sul”. Boletim Economia \& Tecnologia, Ano 02, Vol. 4. CEPEC: UFPR.

BRESSER-PEREIRA, L.C. (2004). Desenvolvimento e Crise no Brasil. São Paulo: Editora 34.

CARVALHO, F.C. (1992). Mr. Keynes and the Post Keynesians: principles of macroeconomics for a monetary production economy. Aldershot: Edward Elgar.

DAVIDSON, P. (1986). "Finance, funding, saving and investment". Journal of Post Keynesian Economics, Vol. IX, N.1.

DUTT, A.K. (2003). "Income elasticities of imports, north-south trade and uneven development" In: Dutt, A.K; Ross, J. (orgs.). Development Macroeconomics and Structuralist Macroeconomics. Aldershot: Edward Elgar.

ENGLE, R.F.; GRANGER, C.W.J.(1987). "Co-integration and error correction: representation, estimation and testing". Econometrica, vol. 55, pp. 251-76.

FRANCO, G.H.B. (1999). O Desafio Brasileiro. São Paulo: Editora 34.

FURTADO, C. (1986). Teoria e Política do Desenvolvimento Econômico. São Paulo: Nova Cultural.

GRANGER, C.W.J. (1981). "Some properties of time series data and their use in econometric model specification". Journal of Econometrics, vol. 16, pp. 121-30.

HAMILTON, J.D. (1994). Time Series Analysis. Princeton: Princeton University Press.

HARROD, R. (1939). "An essay in dynamic theory”. The Economic Journal, vol. 49.

INTERNATIONAL FINANCIAL STATISTICS, www.imfstatistics.org. Acesso em: 10/01/2007.

JOHANSEN, S. (1988). "Statistical analysis of cointegration vectors". Journal of Economic Dynamics and Control, vol. 12, n. 2-3, pp. 231-54.

. (1991). "Estimation and hypothesis testing of cointegrating vectors in Gaussian Vector autoregressive models". Econometrica, vol. 59, n. 6, pp. 1551-80.

;JUSELIUS, K. (1990). "Maximum likelihood estimation and inference on cointegration: with applications to the demand for money". Oxford Bulletin of Economics and Statistics, vol. 52, n. 2, pp. 169-210.

KALDOR, N. (1956). "Alternative theories of distribution". Review of Economic Studies, Vol. XXIII.

. (1957). "A model of economic growth". The Economic Journal, vol. 67, n. 268, pp. 591-624.

. (1988). "The role of effective demand in the short and long-run growth" In: Barrére, A. (org.). The Foundations of Keynesian Analysis. Londres: Macmillan Press.

KWIATKOWSKI, D.; PHILLIPS, P. C. B.; SCHMIDT, P.; SHIN, Y. (1992). “Testing the Null hypothesis of stationary against the alternative of a unit root". Journal of Econometrics, vol. 54, pp. 159-78.

LEDESMA, M.L. (2002). "Accumulation, innovation and catching-up: an extended cumulative growth model”. Cambridge Journal of Economics, Vol. 26, n. 2.

LEDESMA, M.L; THIRLWALL, A. (2002). "The endogeinity of the natural rate of growth". Cambridge Journal of Economics, Vol. 26, n. 4.

MACKINNON, J. (1990). “Critical values for cointegration tests”. Economics Working Paper Series 90-4. San Diego: University of California.

MADDALA, G.S. (2001). Introduction to Econometrics (Third Edition).New Jersey: Wiley \& Sons.

NEWEY, W; KENNETH, W. (1987). "A simple, positive semi-definite, heteroskedastic and auto-correlation consistent covariance matrix”. Econometrica, 55(3), pp.703-708.

OREIRO, J.L. (2000). "O debate entre Keynes e os clássicos sobre os determinantes da taxa de juros: uma grande perda de tempo?”. Revista de Economia Política, Vol. 20, n. 2. 
. (2004). "Accumulation regimes, endogenous desired rate of capacity utilization and income distribution”. Investigación Económica, Vol. LXIII, n. 248.

OREIRO, J.L.C.; SILVA, G.J; FORTUNATO, W. (2008). "Gasto público com infraestrutura, acumulação privada de capital e crescimento de longo prazo: uma avaliação teórica e empírica para o Brasil (1985-2003)”. XIII Encontro Nacional de Economia Política, João Pessoa.

PARK, M.S. (2000). "Autonomous demand and the warranted rate of growth". Metroeconomica.

PARK, H.M. (2003). “Testing Normality in SAS, STATA, and SPSS”. Site: http://pytheas.ucs.indiana. edu. Site acessado em 09/09/2006.

SETTERFIELD, M. (1997). Rapid Growth and Relative Decline. Londres: Macmillan Press.

THIRLWALL, A. (1979). "Okun's law and the natural rate of growth". The Southern Economic Journal, Vol. 36, n. 1.

(2002). The Nature of Economic Growth. Aldershot: Edward Elgar.

TAYLOR, L. (1994). "Gap models". Journal of Development Economics, 45. 